Original Article

\title{
A study of clinical, radiological and etiological profile of cerebral venous sinus thrombosis at a tertiary care center
}

\author{
Col R.K. Anadure, VSM ${ }^{a, *}$, Surg Cdr Vinny Wilson ${ }^{b}$, \\ Gp Capt Samaresh Sahu ${ }^{c}$, Surg Cdr Anuj Singhal ${ }^{d}$, Satish Kota ${ }^{e}$ \\ a Senior Adviser (Medicine \& Neuro), Command Hospital (Air Force), Bengaluru, India \\ ${ }^{\mathrm{b}}$ Classified Specialist (Medicine \& Neuro), INHS Asvini, Mumbai, India \\ ${ }^{\mathrm{c}}$ Senior Adviser (Radiology), INHS Asvini, Mumbai, India \\ d Associate Professor, Department of Medicine, Armed Forces Medical College, Pune 411040, India \\ ${ }^{\mathrm{e}}$ Resident (Medicine), INHS Asvini, Mumbai, India
}

\section{A R T I C L E I N F O}

Article history:

Received 1 April 2017

Accepted 17 November 2017

Available online 16 December 2017

Keywords:

Cerebral venous thrombosis

Hemorrhagic infarct

Venography

Heparin

\begin{abstract}
A B S T R A C T
Background: A prospective, observational study was done at a tertiary care hospital in Western Maharashtra to describe the etiologies, clinical features, diagnosis and prognosis of cerebral venous sinus thrombosis in an Indian population.

Methods: 54 patients with clinical and MRI features suggestive of cerebral venous sinus thrombosis (CVST), were studied with detailed clinical evaluation and pro-thrombotic work up. All were followed up monthly for 6 months, to assess the response to therapy and clinical outcomes.

Results: The mean age of presentation was 35 years. Headache was the most common presenting symptom (94\%) and hemi paresis (22\%) was the most common neurological sign. The most common sinus involved was transverse sinus in $77 \%$ of cases. In the unprovoked CVST subset $(n=29)$, elevated factor VIII $(72 \%)$ and protein C deficiency $(24 \%)$ were the common prothrombotic states identified. In the provoked CVST subset $(n=18)$, puerperium (44\%) and para-infectious (22\%) accounted for majority cases. Idiopathic CVST accounted for $13 \%(7 / 54)$ in this study. A Modified Rankin Scale (MRS) of 0-1 was achieved in $96 \%$ of patients at the end of 6 months follow up with no mortality in this study.

Conclusion: CVST is an important yet under recognized cause of intracranial hypertension and stroke in young. Clinical presentation is extremely varied and a high index of suspicion is needed. Magnetic Resonance Imaging (MRI) brain with Magnetic Resonance Venography (MRV) is the current diagnostic modality of choice. Elevated factor VIII and puerperium are the common etiologies in an Indian population. Management with anticoagulants is safe and has excellent clinical outcomes.
\end{abstract}

○ 2017 Published by Elsevier B.V. on behalf of Director General, Armed Forces Medical Services.

\footnotetext{
* Corresponding author.

E-mail address: ravi.anadure@gmail.com (R.K. Anadure).

https://doi.org/10.1016/j.mjafi.2017.11.004

0377-1237/@ 2017 Published by Elsevier B.V. on behalf of Director General, Armed Forces Medical Services.
} 


\section{Introduction}

Thrombosis of the cerebral veins and dural sinuses is a distinct subgroup of cerebrovascular disease, which is often a diagnostic and therapeutic challenge due to varying and misleading clinical presentations. Cerebral venous sinus thrombosis (CVST) accounts for $10-20 \%$ of stroke in young which in turn accounts for nearly $30 \%$ of all cases of strokes in India. ${ }^{1}$ More than 100 causes of CVST have been recorded in literature. ${ }^{1}$ However even with extensive investigation no cause is identified in $20-25 \%$ of the cases. Pro-coagulant conditions account for most of the primary or unprovoked CVST. Pregnancy and early puerperium are well established risk factors for provoked CVST in India. $^{2}$

Headache is the most common presenting symptom in 70$90 \%$ of cases. Focal deficits such as hemi paresis, seizures, impaired level of consciousness and papilledema can occur in one-third to three-quarters of cases. ${ }^{3}$ The above features are present in various combinations ranging from syndrome of raised intracranial pressure without localization to deep altered sensorium and dense hemiparesis. Neuroimaging is the corner stone in diagnosis of CVST. Magnetic Resonance Imaging (MRI) brain with Magnetic Resonance Venograpphy (MRV) is the current diagnostic modality of choice with very high sensitivity and specificity. ${ }^{4}$

The current therapeutic options for CVST includes antithrombotic treatment with heparin overlapping with oral anticoagulants, thrombolysis (intravenous/local thrombolysis by selective sinus catheterization) and a combination of thrombolysis and anticoagulation. ${ }^{5,6}$ Evaluation for an underlying pro-coagulant state may be rewarding for further prevention with long term anti coagulation. ${ }^{7}$ In addition to this, symptomatic treatment of intracranial hypertension, seizures, headache, visual failure and etiological treatment to manage the associated conditions is essential.

Outcome of CVST is a bit unpredictable, it is not unusual to see dramatic recovery in deeply comatose patient and, sudden worsening in conscious patients due to extension of thrombosis. $^{7}$ Early diagnosis and appropriate treatment of CVST is essential, as it may prevent morbidity and can be life saving.

We therefore attempted to fill the lacunae in our understanding of CVST in an Indian setting, by conducting this study at our referral center. It was aimed at specifically the clinical profile, radiological features, etiological factors and outcome of CVST in patients of venous stroke admitted at a tertiary care center.

\section{Materials and methods}

The study was carried out at a tertiary care multi-specialty hospital in Western Maharashtra. Study protocol was approved by the institutional ethics committee and only patients/NOK who gave a written informed consent were included in the study. The study design was a prospective, observational study with patients recruited over a 20 months period from December 2014 to August 2016.
All patients suspected to have cerebral venous sinus thrombosis based on clinical profile of raised intracranial pressure, seizures, with or without focal neurological deficits and confirmed imaging diagnosis based on MRI of brain with MRV, were included in the study. Written informed consent was obtained from all such patients eligible for the study. Patients were excluded in cases of (i) inconclusive neuroimaging, (ii) arterial strokes, (iii) space occupying lesions, (iv) metabolic encephalopathy, and (v) patient unwilling to participate.

Meticulous history, clinical examination, laboratory investigations were undertaken in all cases of cerebral venous sinus thrombosis as per a detailed Performa. Those who presented within $48 \mathrm{~h}$ were considered to have acute onset, with onset longer than $48 \mathrm{~h}$ but less than 1 month were considered sub acute onset, and those with presentation more than 1 month after symptoms as chronic onset. Hazardous alcohol use (WHO working definition ${ }^{8}$ - regular average consumption of 20-40 g/ day for women and 40-60 g/day for men) was also specifically recorded in all cases, as it has been found to be a risk factor for venous strokes.

All the patients underwent MRI and MRV of brain on a 1.5 T MRI system (Symphony; Siemens; Erlangen, Germany). The scan protocol included an axial Fluid Attenuated Inversion Recovery (FLAIR), axial and coronal T2W, T1 3D sagittal, diffusion weighted at $B$ value of 0.500 and 1000 s/Sq mm with generation of Apparent Diffusion Coefficient (ADC) map, axial gradient sequence. This was followed by contrast MRV after injecting $20 \mathrm{ml}$ of gadolinium (Magnavist; Bayer Zydus) using a pressure injector at a rate of $4 \mathrm{ml} / \mathrm{s}$ and acquiring repeated continuous image of the brain. The images so obtained were subtracted from the non contrast images. 3D MR venogram was obtained after post processing the base images, using dedicated software on a workstation.

All patients were treated with standard institutional protocols of anticoagulation (5-7 days) of Low Molecular Weight Heparin (LMWH) followed by 3-5 days overlap with oral anticoagulants to achieve Internationally Normalized Ratio (INR) in range 2-3. Anti epileptics, osmotic diuretics and other supportive therapy were used wherever required. All patients were followed up for a period of 6 months at a monthly interval to assess the response to therapy and clinical outcomes. A pro-coagulant workup was done only in unprovoked CVST cases at 3 months after the ictus. Blood samples were drawn 2 weeks after stopping Oral Anticoagulants (OAC) and switching to LMWH. The work up included a standard testing panel of factor VIII, Protein C \& S, Anti Phospholipid Antibodies (APLA) panel and anti-thrombin III. No genetic tests were carried out due to financial constraints. The flow of patients into the study, with their exclusions at various levels, and the final outcomes is as shown in the consort diagram (Fig. 1).

\section{Statistical analysis}

The data thus collected was entered into Microsoft Excel work sheets. Analysis of the data was carried out using appropriate descriptive statistics. 


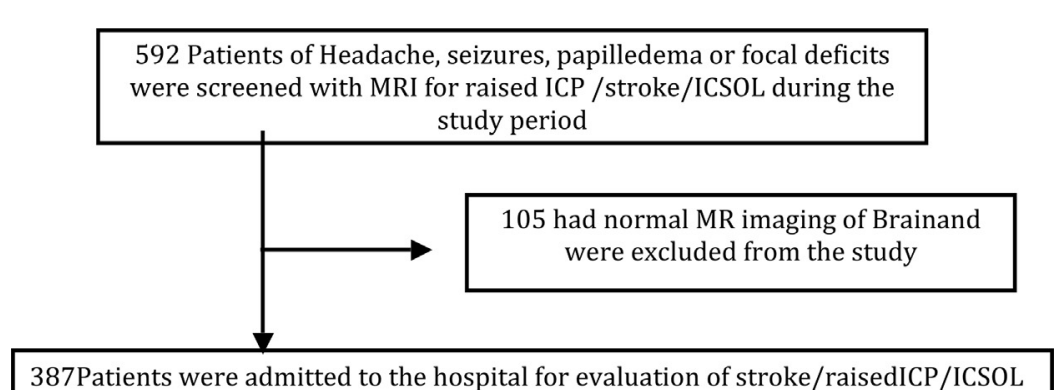

387Patients were admitted to the hospital for evaluation of stroke/raisedICP/ICSOL

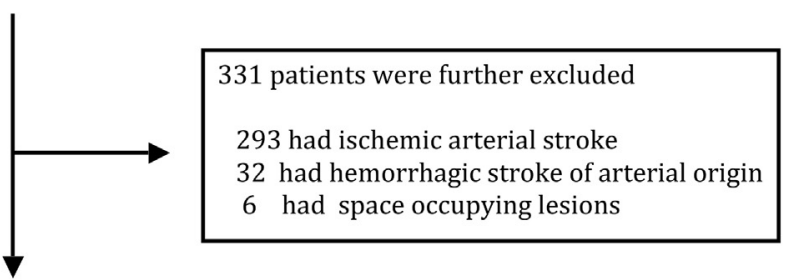

56 Patients with confirmed CVST on MRI were included in the study

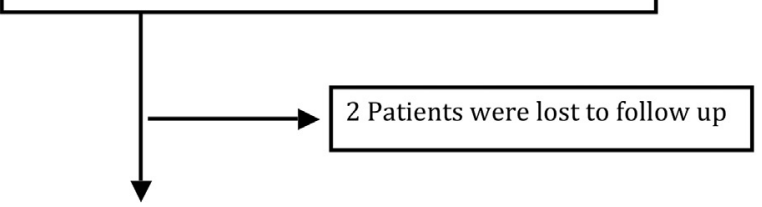

54 Patients completed the study and were followed up for 6 months at monthly intervals

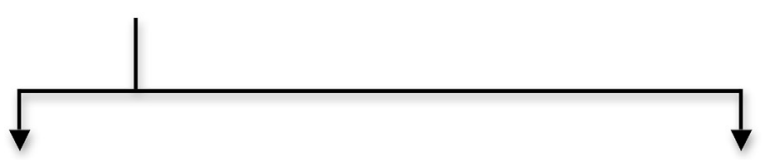

Unprovoked CVST $(n=36)$

Provoked CVST $(n=18)$

Fig. 1 - Consort diagram showing flow of patients in the study period.

\section{Results}

\section{Clinical profile (details as depicted in Table 1)}

A total of 54 patients were included in this study, 39 (72\%) were males and 15 (28\%) were females. The mean age of presentation was $35 \pm 4$ years. Most of the patients had sub acute presentation (53\%), followed by acute (29\%) and chronic onset $(18 \%)$. Headache was the most common presenting symptom (94\%) followed by vomiting (62\%), seizures (42\%) and focal deficits (33\%). Altered sensorium was noted in $32 \%$ of cases. Fever was present in $15 \%$ of cases at the onset. Hemiparesis (22\%) was the common neurological sign followed by papilledema (18\%). Cranial nerve palsies were seen in $7 / 54$ (12\%) of cases, which included Upper Motor Neuron (UMN) seventh nerve palsy in $6 / 7$ cases and bilateral sixth nerve paresis in 1 patient.
Radiologic features: (details as depicted in Table 2)

Magnetic Resonace Imaging (MRI) brain and contrast MRV are most useful in the diagnosis of cerebral venous thrombosis. In the T2W images there will be absence of flow voids in the effected venous sinuses (black arrows in Fig. 2). When this is associated with an infarct which does not follow the conventional arterial territory, it is the classical imaging description of venous infarct in cerebral venous thrombosis (CVT). The cortical veins if thrombosed, appear as hypointense cord like structures on the cortical surface, in T2W and gradient images. Contrast MR venography classically reveals non filling of the effected cortical venous sinuses (white arrows in Fig. $3 a$ and b). Post contrast T1W images shows filling defect in the thrombosed sinuses (black arrow in Fig. 4).

In the present study, 37 cases (68\%) had venous infarctions on MRI brain, out of which 26 cases (48\%) were hemorrhagic infarctions and 11 cases (20\%) were non-hemorrhagic infarc- 
Table 1 - Clinical profile of cerebral venous sinus thrombosis in study group.

\begin{tabular}{lcc} 
Symptoms and signs & No. of patients & Percentage \\
\hline Headache & 51 & 94 \\
Vomiting & 33 & 62 \\
Seizure & 23 & 42 \\
Altered sensorium & 17 & 32 \\
Limb weakness & 12 & 22 \\
Fever & 8 & 15 \\
Hemiparesis & 12 & 22 \\
Pallor & 13 & 24 \\
Papilledema & 9 & 16 \\
Cranial nerve involvement & 7 & 12 \\
Dysarthria/aphasia & 6 & 11 \\
\hline
\end{tabular}

\section{Table 2 - Sinuses involved on MRV in the study group.}

\begin{tabular}{lcc} 
Sinus involved & No. of patients & Percentage \\
\hline Transverse sinus (TS) & 40 & 74 \\
Superior sagittal sinus (SSS) & 29 & 52 \\
Sigmoid sinus (SS) & 27 & 50 \\
Straight sinus & 4 & 7 \\
Cortical veins & 2 & 4 \\
Deep veins & 1 & 2 \\
\hline
\end{tabular}

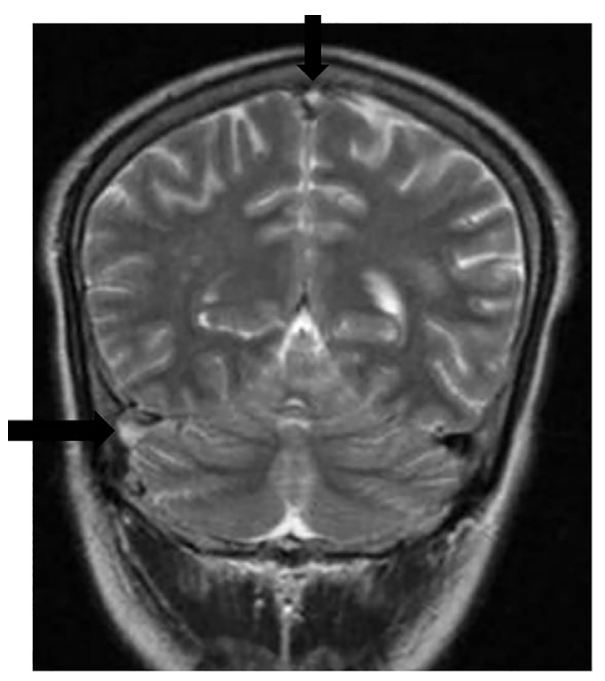

Fig. 2 - T2W coronal images shows loss of normal flow voids (black arrows) in the right transverse and superior sagittal sinus suggestive of CVT.

tions in a venous distribution. The remaining 17 (32\%) cases had only dural or cortical CVT without parenchymal lesions. Most frequently involved sinus was transverse sinus in $77 \%$ (42/54), followed by superior sagittal sinus in 57\% (31/54), sigmoid sinus in 53\% (29/54), straight sinus in $8 \%$ (4/54), cortical veins alone in 5\% (3/54) and deep veins in 2\% (1/54). A Digital Subtraction Angiography (DSA) for confirmation of diagnosis was needed in only $3 / 54(5 \%)$ cases, who had pure cortical CVT with normal dural sinuses. A therapeutic DSA with intra-dural thrombolytic was needed in only $1 / 54$ cases.

\section{Etiology: (details as depicted in Tables 3 and 4)}

From an etiological point of view, 18/54 (33\%) had clear clinical triggers and were considered provoked CVT in this study. In this subset, para infectious $8 / 18$ (44\%), puerperium $4 / 18(22 \%)$, high altitude exposures 4/18 (22\%) and OCP use 2/18 (11\%) were identified as risk factors for provoking CVT. The breakdown of para infectious CVT included mastoiditis (3 patients), viral meningo-encephalitis (2 patients), Ebstine Barr Virus (EBV) infection (1 patient), Dengue fever (1 patient) and Varicella zoster (1 patient). In the other subset of unprovoked CVT 36/54 (67\%) where no clinical triggers were evident, a standard procoagulant workup was done. In 29 (80\%) of these cases a prothrombotic state could be identified. This included Elevated Factor VIII in 21/36 (58\%), Protein C deficiency in 9/36 (25\%), Protein S deficiency in 5/36 (14\%) and APLA in 1/36 (3\%). Some patients had combined pro-thrombotic states, like elevated Factor VIII and Protein C deficiency in 5 cases, and both Protein $\mathrm{C} \& \mathrm{~S}$ deficiency in 2 cases. None of the patients showed Anti Thrombin III deficiency. In the remaining 7/54 (13\%) no clear clinical or lab abnormality could be identified and these were considered Idiopathic subset in this study. They may have genetic prothrombotic states like FVL (Factor V Leiden) or MTHFR (methylenetetrahydrofolate reductase) which were not tested for in this study. Hazardous alcohol use (as per WHO working definition) was noted in 19/54 (35\%). Of these alcohol consumers, $16 / 19$ patients had some form of thrombophilia, and $3 / 19$ had provoked CVST. This strongly suggests that, alcohol abuse seems to act as a precipitating factor in the pathogenesis of CVT, in the presence of other prothrombotic states or clinical triggers.

\section{Treatment and prognosis}

All patients were treated with low molecular weight heparin (Inj Enoxaparin $1 \mathrm{mg} / \mathrm{kg}$ SC twice daily) for 5-7 days followed by overlap with oral anti-coagulants for 3-5 days, till target INR of 2-3 was achieved. Anti epileptics and osmotic diuretics were used wherever required. All patients showed very good response to treatment with 34/54 (62\%) achieving complete recovery and a Modified Rankin Scale (MRS) - 0 (No symptoms at all) at 6 months follow up. Another 18/54 (33\%) had near complete recovery with MRS - 1 (No significant disability despite symptoms) at 6 months. Only 2/54 (4\%) had slight disability with MRS -2 at the end of 6 months follow up. Only one patient with extensive dural thrombosis and resistance to LMWH at second week of therapy, needed DSA and intra-dural thrombolysis of SSS and TS. He subsequently made a good clinical recovery (MRS-2). There were no deaths in the entire study population.

\section{Discussion}

Cerebral venous sinus thrombosis, unlike arterial stroke, often occurs in young individuals. ${ }^{1}$ It is often a diagnostic challenge due to varying and misleading clinical presentation. However, 


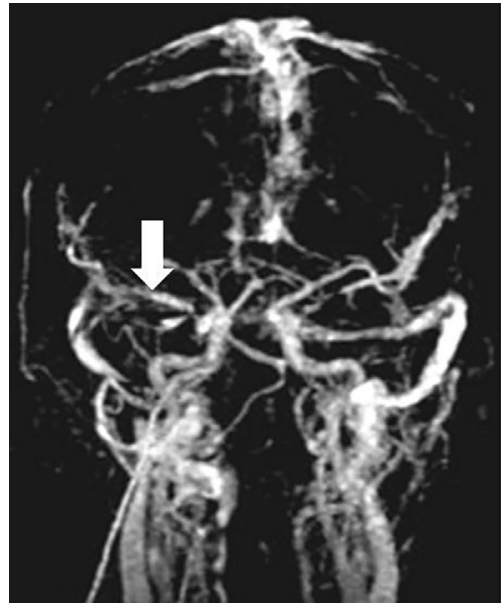

a

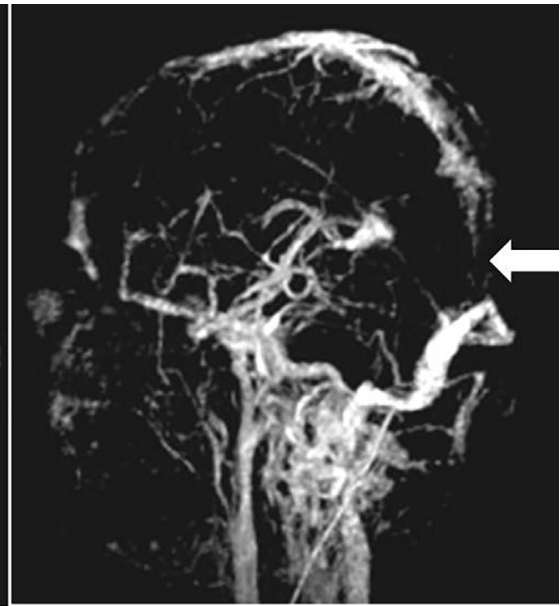

b

Fig. 3 - ( $a$ and $b$ ) Maximum intensity projections MR venography images reveals thrombosis of the right transverse (white arrow in a) and superior sagittal sinus (white arrow in b).

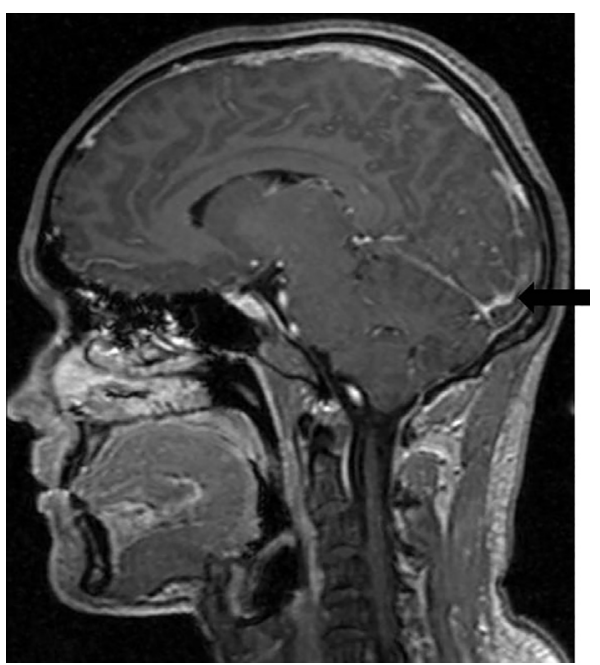

Fig. 4 - Post contrast T1WI shows large filling defect in the superior sagittal sinus (black arrow).

Table 3 - Etiologic profile of cerebral venous thrombosis in the study.

\begin{tabular}{lcc} 
Risk factors & No. of patients & Percentage \\
\hline Provoked (18/54) & & \\
Parainfectious & 8 & 44 \\
High altitude exposure & 4 & 22 \\
Pregnanacy/puerperium & 4 & 22 \\
OCP use & 2 & 11 \\
Unprovoked (36/54) & & \\
$\quad$ Procoagulant conditions & 29 & 80 \\
Idiopathic & 7 & 19 \\
\hline
\end{tabular}

modern neuro-imaging techniques, diagnostic laboratory investigations, and genetics are providing more information about risk factors and clinical profile of CVST. We compared
Table 4 - Pro-coagulant conditions identified in unprovoked CVST.

\begin{tabular}{lcc}
$\begin{array}{l}\text { Procoagulant } \\
\text { conditions }(n=36)\end{array}$ & $\begin{array}{c}\text { No. of } \\
\text { patients }\end{array}$ & Percentage \\
\hline Factor VIII elevation & 21 & 58 \\
Protein C deficiency & 9 & 25 \\
Protein S deficiency & 5 & 14 \\
APLA panel & 1 & 3 \\
At III deficiency & Nil & 0 \\
Combined factor VIII \& Prot C & 5 & 14 \\
Combined Prot C \& S Def & 2 & 6 \\
\hline
\end{tabular}

our experience of CVST, highlighting its diverse clinical presentations, predisposing factors, and neuro-imaging with other studies from India and the west.

Comparing the age group involved, 20-40 years was the commonest age group involved in various studies with mean age of onset being 35 years. The present study also showed similar finding with mean age of onset being $34 \pm 4$ years. Our study showed male preponderance (M:F - 2.6:1), as the study was done at tertiary care center related to Armed forces (the study population contains predominately males).

Headache was the most common symptom in the present study accounting for $94 \%$ of patients. Sudden onset severe headache with the focal deficits was seen in $8 \%$ cases, and without focal deficits in $10 \%$ of patients. New onset sub acute and focal headaches ( $<4$ weeks duration) associated with recurrent vomiting (36\%) or focal deficits (34\%), strongly suggested the diagnosis of CVST in this study. Chronic headache ( $>4$ weeks duration) was seen in $16 \%$ of patients who were initially misdiagnosed as migraine or tension headache, and later due to increased in frequency and severity of headaches underwent MRV which revealed CVST. Most common pattern of headache was diffuse throbbing type (56\%) followed by dull aching type (26\%). Focal headache were seen in $36 \%$ of cases and site of headache correlated with site of thrombosis in $58 \%$ of them. 
Seizures are far more frequently seen in CVST than in arterial stroke. At times seizures are heralding symptoms in CVT and should arouse the suspicion of the diagnosis. In our study, $42 \%$ of cases had seizures which is comparable with Narayan et al., ${ }^{2}$ and most other western studies. Seizure as the initial presentation was seen in 3 patients (5\%) in this study. The types of the seizures observed in the patients were generalized tonic-clonic (65\%), focal with or without dyscognitive features (25\%) and focal with secondary generalization (10\%).

$32 \%$ patients had altered level of consciousness at presentation, which is comparable with Wasay et al. ${ }^{10}$ and Ameri et al. ${ }^{11}$ The cause of altered sensorium can be attributed to post-ictal states, raised Intra Cranial Pressure (ICP), aphasia, deep CVT and delirium due to viral meningo-encephalitis. 15\% patients had fever at the onset which is similar to findings of Wasay et al. ${ }^{10}$ and Anadure et al. ${ }^{12}$

Prothrombotic conditions are the most common risk factor identified for unprovoked CVST in published literature throughout the world. In the International study on Cerebral Venous Thrombosis (ISCVT) cohort, ${ }^{9} 34 \%$ patients had prothrombotic conditions, and $22 \%$ had underlying genetic prothrombotic states. However earlier published studies from India did not have information regarding these prothrombotic conditions due to paucity of laboratory data. Recently Pai et al. ${ }^{7}$ and Narayan et al., ${ }^{2}$ reported thrombophilia as a risk factor for CVST in $18 \%$ and $12.3 \%$ patients respectively. In our study 29 / $36(80 \%)$ of the unprovoked CVST patients had predisposing thrombophilic conditions. Plasma Factor VIII was elevated in $21 / 36(58 \%)$ patients tested. Protein C deficiency was noted in 9/ $36(25 \%)$ and Protein S deficiency in 5/36 (19\%) patients tested. All are well-established lab phenotypes that predispose to venous thrombosis. Factor VIII elevation has been implicated as independent risk factor for CVST in Indians. ${ }^{11}$ Majority of cases of CVST as recorded in published literature, have multifactorial etiology, which suggests that pro-thrombotic workup should be complete and exhaustive. However, this is not practical in all centers due to economic reasons.

Nagaraja et al., ${ }^{13}$ had found that 200 out of 230 cases (86\%) of CVST, seen over eight years at a tertiary neurology facility, were puerperal in nature. The experience of other authors from India has been similar like $\mathrm{Neki}^{14}$ had found $62 \%$ of their cases of CVT in happened in postpartum period. In the present study, puerperal group consists of only $4 / 15$ (26\%). The decreased incidence in this study is attributed to a population bias, as the study center caters serving and retired Armed forces personnel, majority of who are males. Further the improved and meticulous obstetric care with $100 \%$ institutional deliveries, may have brought down the rates of puerperal CVT.

Alcohol use is common in Armed forces. Even those people who drink in moderation can still encounter alcohol related problems. In our study Hazardous alcohol use was seen in 35\% $(16 / 54)$ of cases. Among these cases, 84\% (16/19) patients had underlying thrombophilia. Alcohol seems to acts as a potentiating agent in the presence of other thrombogenic factors for CVST, in our study population.

In $13 \%$ (7/54) of cases we were unable to find any cause even after procoagulant workup, this constituted Idiopathic cases in our study. They may be harboring some form of genetic thrombophillia like FVL or MTHFR mutation, but we did not test for the same due to financial constraints.

In the International study on cerebral vein and dural sinus thrombosis, the reported frequency of involvement of various sinuses was, superior sagittal sinus (62\%), transverse sinus (42\%), straight sinus (18\%), cortical veins (17.1\%) and deep veins $(10.9 \%)$. In the present study transverse sinus was most frequently involved (40/54) 74\% followed by superior sagittal sinus in (29/54) 52\%, sigmoid sinus in (27/54) 50\%, Straight sinus in (4/54) $7 \%$, cortical veins alone in $(2 / 54) 4 \%$ and deep veins in (1/50) $2 \%$ which is comparable with Bousser et al. ${ }^{15}$ The most common MRI finding was hemorrhagic venous infarction accounting for $30 \%$ of cases. Similar observations were noted in a recent study by Banakar et al. ${ }^{16}$ Table 5 compares the clinical, radiological and etiological profile of this study, with other large Indian cohort studies on CVT.

In the Armed Forces, previous studies by Kumaravelu et al. ${ }^{17}$ and Mehta et al., ${ }^{18}$ have reported high altitude exposure as a very important provoking factor peculiar to the troops deployed in these areas. The manifestations can be very subtle and protean, requiring a high index of suspicion toward CVT, for doctors working in these regions. Lowering the altitude and early treatment with heparin seems to have good clinical outcomes. In the present study $4 / 18$ (22\%) patients in the provoked CVT subset had recent High altitude exposure, and responded very well to LMWH therapy.

In the past CVST had been associated with a dismal prognosis and high mortality rates. The recent ISCVT study, performed in the era of modern neuroimaging, LHWH

Table 5 - Comparison of present study with similar Indian cohort studies on CVST.

\begin{tabular}{|c|c|c|c|c|c|c|}
\hline & $\begin{array}{c}\text { Parikh } \\
\text { et al., }{ }^{19}(1987) \\
(n=110)\end{array}$ & $\begin{array}{c}\text { Narayan } \\
\text { et al., }{ }^{2}(2012) \\
(n=428)\end{array}$ & $\begin{array}{c}\text { Pai } \\
\text { et al., }{ }^{7}(2013) \\
(n=612)\end{array}$ & $\begin{array}{c}\text { Anadure } \\
\text { et al., }{ }^{12}(2014) \\
(n=48)\end{array}$ & $\begin{array}{c}\text { Aneesh } \\
\text { et al.., }{ }^{20}(2017) \\
(n=116)\end{array}$ & $\begin{array}{l}\text { Present } \\
\text { study } \\
(n=54)\end{array}$ \\
\hline Mean age (years) & 34.6 & 31.3 & 31.9 & 30.9 & 35.2 & 34.4 \\
\hline Male:female & $2: 1$ & $1.16: 1$ & 3:2 & $1.18: 1$ & $1.32: 1$ & $2.6: 1$ \\
\hline Headache & $38.1 \%$ & $88.3 \%$ & $61.9 \%$ & $98 \%$ & $62 \%$ & $94 \%$ \\
\hline Seizure & $60 \%$ & $39.9 \%$ & $31.2 \%$ & $59 \%$ & $51.7 \%$ & $40 \%$ \\
\hline Hemiparesis & $38.1 \%$ & $25.3 \%$ & $47.7 \%$ & $41 \%$ & $20.6 \%$ & $22 \%$ \\
\hline Papilledema & $29 \%$ & $63.4 \%$ & $62.4 \%$ & $69 \%$ & $2.58 \%$ & $16 \%$ \\
\hline Sinus involved (most common) & NA & NA & NA & $\begin{array}{l}\text { Superior sagittal } \\
\text { Sinus (SSS) } \\
(49 \%)\end{array}$ & $\begin{array}{l}\text { Superior sagittal } \\
\text { Sinus (SSS) } \\
(50.8 \%)\end{array}$ & $\begin{array}{l}\text { Transverse } \\
\text { Sinus (TS) } \\
(74 \%)\end{array}$ \\
\hline Thrombophilia & NA & $12.3 \%$ & $18 \%$ & $72 \%$ & NA & $53 \%$ \\
\hline
\end{tabular}


administration, and endovascular intervention, reported much lower mortality rates (8-14\%) and significantly better outcome. ${ }^{8}$ In our study all the 54 patients were treated with LMWH followed by oral anticoagulants. All patients showed good response to treatment with no mortality in the entire study period, and $96 \%$ had near complete recovery at 6 months achieving a MRS of $0-1$. This rather dramatic outcome may be attributed to free comprehensive health care for members of Armed Forces, with easy access to Neurology and MRI services, leading to early diagnosis and prompt initiation of appropriate treatment with anti-coagulants.

\section{Conclusion}

Cerebral Venous Sinus Thrombosis (CVST) still remains an uncommon cause of headache and stroke, but modern MR imaging has allowed early and firm diagnosis. It is one of the treatable and reversible causes of stroke in young. Clinical presentation is extremely varied and symptoms may evolve over few weeks or even months. Important clinical features to suggest this disorder are, new onset of focal headache, or headache onset with seizures, papilledema or focal deficits, in the appropriate clinical settings. Evaluation for an underlying procoagulant in unprovoked CVT, is useful for further planning long term anti coagulation. The newly defined entity of elevated Factor VIII was one of the most common pro coagulant conditions identified in this study population. Management with heparin (LMWH), overlapping with oral anti coagulation showed optimum response to therapy, with excellent clinical outcomes. The present study identifies subtle clinical markers and new prothrombotic states, to allow early suspicion and diagnosis of CVST, which in turn leads to excellent long-term outcomes.

\section{Limitations of the study}

1) This study was conducted in a closed group of population which included Armed forces personnel and their dependents only (study population contained predominately males (M:F - 2.6:1)).

2) A complete thrombophilia evaluation could not be performed in every patient due to financial constraints. This could have helped in understanding complex interplay of these factors, in both provoked and unprovoked subsets.

\section{Conflicts of interest}

The authors have none to declare.

\section{Acknowledgements}

Faculty and staff at Dept of Medicine \& Radilogy, INHS Asvini.

\section{R E F E R E N C E S}

1. Dash D, Prasad K, Joseph L. Cerebral venous thrombosis: an Indian perspective. Neurol India. 2015;63:318-328.

2. Narayan D, Kaul S, Ravishankar K, et al. Risk factors, clinical profile and long term outcome of 428 patients of cerebral sinus venous thrombosis: insights from Nizam's Institute Venous Stroke Registry, Hyderabad (India). Neurol India. 2012;60:154-159.

3. Current concepts: thrombosis of the cerebral veins and sinuses. N Engl J Med. 2005;352:1791-1798.

4. Connor SE, Jarsoz JM. Magnetic resonance imaging of cerebral venous sinus thrombosis. Clin Radiol. 2002;57:449-546.

5. Misra UK, Kalita J, Chandra S, et al. Low molecular weight heparin versus unfractionated heparin in cerebral venous sinus thrombosis: a randomized controlled trial. Eur J Neurol. 2012;19:1030-1036.

6. Dentali F, Squizzato A, Gianni M, et al. Safety of thrombolysis in cerebral venous thrombosis. A systematic review of the literature. Thromb Haemost. 2010;104:1055-1062.

7. Pai N, Ghosh K, Shetty S. Hereditary thrombophilia in cerebral venous thrombosis: a study from India. Blood Coagul Fibrinolysis. 2013;24:540-543.

8. World Health Organization. The ICD-10 Classification of Mental and Behavioural Disorders: Clinical Descriptions and Diagnostic Guidelines. Geneva: WHO; 1992.

9. Ferro JM, Canhao P, Stam J, Bousser MG, Barinagarrementeria F, for the ISCVT investigators. Prognosis of cerebral vein and dural sinus thrombosis: results of the International Study on Cerebral Vein and Dural Sinus Thrombosis (ISCVT). Stroke. 2004;35:664-670.

10. Wasay M, Bakshi R, Bobustuc G, et al. Cerebral venous thrombosis: analysis of a multicenter cohort from the United States. J Stroke Cerebrovasc Dis. 2008;17:49-54.

11. Ameri A, Bousser MG. Cerebral venous thrombosis. Neurol Clin. 1992;10:87-111.

12. Anadure RK, Nagaraja D, Christopher R, et al. Plasma factor VIII in non-puerperal cerebral venous thrombosis: a prospective case-control study. J Neurol Sci. 2014;339 (1):140-143.

13. Nagaraja D, Sarma GR. Treatment of cerebral sinus/venous thrombosis. Neurol India. 2002;50:114-116.

14. Neki NS. Clinical profile of cortical vein thrombosis - a two years experience. Ann Indian Acad Neurol. 2004;7:45-50.

15. Bousser MG, Ferro JM. Cerebral venous thrombosis: an update. Lancet Neurol. 2007;6:162-170.

16. Banakar B, Hiregoudar V. Clinical profile, outcome, and prognostic factors of cortical venous thrombosis in a tertiary care hospital, India. J Neurosci Rural Pract. 2017;8(2):204-208.

17. Kumaravelu S, Aditya A, Singh KK. Cerebral venous thrombosis. Med J Armed Forces India. 2008;64(4):355-360.

18. Mehta SR, Muthukrishnan J, Varadarajulu R. Cerebral venous sinus thrombosis: a great masquerader. Med J Armed Forces India. 2004;60(3):299-301.

19. Parikh PM, Sukthankar RU, Parikh A, et al. Cerebral venous thrombosis. J Assoc Physicians India. 1987;35:349-351.

20. Aneesh T, Gururaj H, Arpitha JS, et al. Clinical features, predisposing factors and radiological study of cerebral venous sinus thrombosis: experience from a tertiary care center in Southern India. Int J Res Med Sci. 2017;5:3023-3028. 